\title{
Basic Properties of Circulant Matrices and Anti-Circular Matrices
}

\author{
Xiaopeng Yue \\ Xuchang University \\ Henan, China
}

\author{
Xiquan Liang \\ Qingdao University of Science \\ and Technology \\ China
}

Summary. This article introduces definitions of circulant matrices, lineand column-circulant matrices as well as anti-circular matrices and describes their main properties.

MML identifier: MATRIX16, version: $\underline{7.9 .03 \quad 4.108 .1028}$

The articles [6], [9], [4], [10], [1], [14], [13], [2], [5], [8], [12], [11], [3], and [7] provide the notation and terminology for this paper.

\section{Some Properties of Circulant Matrices}

For simplicity, we adopt the following convention: $i, j, k, n, l$ denote elements of $\mathbb{N}, K$ denotes a field, $a, b, c$ denote elements of $K, p, q$ denote finite sequences of elements of $K$, and $M_{1}, M_{2}, M_{3}$ denote square matrices over $K$ of dimension $n$.

Next we state two propositions:

(1) $\mathbf{1}_{K} \cdot p=p$

(2) $\left(-\mathbf{1}_{K}\right) \cdot p=-p$.

Let $K$ be a set, let $M$ be a matrix over $K$, and let $p$ be a finite sequence. We say that $M$ is line circulant about $p$ if and only if:

(Def. 1) $\operatorname{len} p=$ width $M$ and for all natural numbers $i, j$ such that $\langle i, j\rangle \in$ the indices of $M$ holds $M_{i, j}=p(((j-i) \bmod$ len $p)+1)$.

Let $K$ be a set and let $M$ be a matrix over $K$. We say that $M$ is line circulant if and only if: 
(Def. 2) There exists a finite sequence $p$ of elements of $K$ such that len $p=$ width $M$ and $M$ is line circulant about $p$.

Let $K$ be a non empty set and let $p$ be a finite sequence of elements of $K$. We say that $p$ is first-line-of-circulant if and only if:

(Def. 3) There exists a square matrix over $K$ of dimension len $p$ which is line circulant about $p$.

Let $K$ be a set, let $M$ be a matrix over $K$, and let $p$ be a finite sequence. We say that $M$ is column circulant about $p$ if and only if:

(Def. 4) $\operatorname{len} p=\operatorname{len} M$ and for all natural numbers $i, j$ such that $\langle i, j\rangle \in$ the indices of $M$ holds $M_{i, j}=p(((i-j) \bmod \operatorname{len} p)+1)$.

Let $K$ be a set and let $M$ be a matrix over $K$. We say that $M$ is column circulant if and only if:

(Def. 5) There exists a finite sequence $p$ of elements of $K$ such that len $p=\operatorname{len} M$ and $M$ is column circulant about $p$.

Let $K$ be a non empty set and let $p$ be a finite sequence of elements of $K$. We say that $p$ is first-column-of-circulant if and only if:

(Def. 6) There exists a square matrix over $K$ of dimension len $p$ which is column circulant about $p$.

Let $K$ be a non empty set and let $p$ be a finite sequence of elements of $K$. Let us assume that $p$ is first-line-of-circulant. The functor LCirc $p$ yields a square matrix over $K$ of dimension len $p$ and is defined by:

(Def. 7) LCirc $p$ is line circulant about $p$.

Let $K$ be a non empty set and let $p$ be a finite sequence of elements of $K$. Let us assume that $p$ is first-column-of-circulant. The functor CCirc $p$ yielding a square matrix over $K$ of dimension len $p$ is defined by:

(Def. 8) CCirc $p$ is column circulant about $p$.

Let $K$ be a field. One can verify that there exists a finite sequence of elements of $K$ which is first-line-of-circulant and first-column-of-circulant.

Let us consider $K, n$. Observe that $0_{K}^{n \times n}$ is line circulant and column circulant.

Let us consider $K$, let us consider $n$, and let $a$ be an element of $K$. Observe that $(a)^{n \times n}$ is line circulant and $(a)^{n \times n}$ is column circulant.

Let us consider $K$. Note that there exists a matrix over $K$ which is line circulant and column circulant.

In the sequel $D$ denotes a non empty set, $t$ denotes a finite sequence of elements of $D$, and $A$ denotes a square matrix over $D$ of dimension $n$.

We now state a number of propositions:

(3) If $A$ is line circulant and $n>0$, then $A^{\mathrm{T}}$ is column circulant.

(4) If $A$ is line circulant about $t$ and $n>0$, then $t=\operatorname{Line}(A, 1)$. 
(5) If $A$ is line circulant and $\langle i, j\rangle \in \operatorname{Seg} n \times \operatorname{Seg} n$ and $k=i+1$ and $l=j+1$ and $i<n$ and $j<n$, then $A_{i, j}=A_{k, l}$.

(6) If $M_{1}$ is line circulant, then $a \cdot M_{1}$ is line circulant.

(7) If $M_{1}$ is line circulant and $M_{2}$ is line circulant, then $M_{1}+M_{2}$ is line circulant.

(8) If $M_{1}$ is line circulant and $M_{2}$ is line circulant and $M_{3}$ is line circulant, then $M_{1}+M_{2}+M_{3}$ is line circulant.

(9) If $M_{1}$ is line circulant and $M_{2}$ is line circulant, then $a \cdot M_{1}+b \cdot M_{2}$ is line circulant.

(10) If $M_{1}$ is line circulant and $M_{2}$ is line circulant and $M_{3}$ is line circulant, then $a \cdot M_{1}+b \cdot M_{2}+c \cdot M_{3}$ is line circulant.

(11) If $M_{1}$ is line circulant, then $-M_{1}$ is line circulant.

(12) If $M_{1}$ is line circulant and $M_{2}$ is line circulant, then $M_{1}-M_{2}$ is line circulant.

(13) If $M_{1}$ is line circulant and $M_{2}$ is line circulant, then $a \cdot M_{1}-b \cdot M_{2}$ is line circulant.

(14) If $M_{1}$ is line circulant and $M_{2}$ is line circulant and $M_{3}$ is line circulant, then $\left(a \cdot M_{1}+b \cdot M_{2}\right)-c \cdot M_{3}$ is line circulant.

(15) If $M_{1}$ is line circulant and $M_{2}$ is line circulant and $M_{3}$ is line circulant, then $a \cdot M_{1}-b \cdot M_{2}-c \cdot M_{3}$ is line circulant.

(16) If $M_{1}$ is line circulant and $M_{2}$ is line circulant and $M_{3}$ is line circulant, then $\left(a \cdot M_{1}-b \cdot M_{2}\right)+c \cdot M_{3}$ is line circulant.

(17) If $A$ is column circulant and $n>0$, then $A^{\mathrm{T}}$ is line circulant.

(18) If $A$ is column circulant about $t$ and $n>0$, then $t=A_{\square, 1}$.

(19) If $A$ is column circulant and $\langle i, j\rangle \in \operatorname{Seg} n \times \operatorname{Seg} n$ and $k=i+1$ and $l=j+1$ and $i<n$ and $j<n$, then $A_{i, j}=A_{k, l}$.

(20) If $M_{1}$ is column circulant, then $a \cdot M_{1}$ is column circulant.

(21) If $M_{1}$ is column circulant and $M_{2}$ is column circulant, then $M_{1}+M_{2}$ is column circulant.

(22) If $M_{1}$ is column circulant and $M_{2}$ is column circulant and $M_{3}$ is column circulant, then $M_{1}+M_{2}+M_{3}$ is column circulant.

(23) If $M_{1}$ is column circulant and $M_{2}$ is column circulant, then $a \cdot M_{1}+b \cdot M_{2}$ is column circulant.

(24) Suppose $M_{1}$ is column circulant and $M_{2}$ is column circulant and $M_{3}$ is column circulant. Then $a \cdot M_{1}+b \cdot M_{2}+c \cdot M_{3}$ is column circulant.

(25) If $M_{1}$ is column circulant, then $-M_{1}$ is column circulant.

(26) If $M_{1}$ is column circulant and $M_{2}$ is column circulant, then $M_{1}-M_{2}$ is column circulant. 
(27) If $M_{1}$ is column circulant and $M_{2}$ is column circulant, then $a \cdot M_{1}-b \cdot M_{2}$ is column circulant.

(28) Suppose $M_{1}$ is column circulant and $M_{2}$ is column circulant and $M_{3}$ is column circulant. Then $\left(a \cdot M_{1}+b \cdot M_{2}\right)-c \cdot M_{3}$ is column circulant.

(29) Suppose $M_{1}$ is column circulant and $M_{2}$ is column circulant and $M_{3}$ is column circulant. Then $a \cdot M_{1}-b \cdot M_{2}-c \cdot M_{3}$ is column circulant.

(30) Suppose $M_{1}$ is column circulant and $M_{2}$ is column circulant and $M_{3}$ is column circulant. Then $\left(a \cdot M_{1}-b \cdot M_{2}\right)+c \cdot M_{3}$ is column circulant.

(31) If $p$ is first-line-of-circulant, then $-p$ is first-line-of-circulant.

(32) If $p$ is first-line-of-circulant, then $\operatorname{LCirc}(-p)=-\operatorname{LCirc} p$.

(33) Suppose $p$ is first-line-of-circulant and $q$ is first-line-of-circulant and len $p=\operatorname{len} q$. Then $p+q$ is first-line-of-circulant.

(34) If len $p=\operatorname{len} q$ and $p$ is first-line-of-circulant and $q$ is first-line-ofcirculant, then $\operatorname{LCirc}(p+q)=\operatorname{LCirc} p+\operatorname{LCirc} q$.

(35) If $p$ is first-column-of-circulant, then $-p$ is first-column-of-circulant.

(36) For every finite sequence $p$ of elements of $K$ such that $p$ is first-columnof-circulant holds $\operatorname{CCirc}(-p)=-\operatorname{CCirc} p$.

(37) Suppose $p$ is first-column-of-circulant and $q$ is first-column-of-circulant and len $p=\operatorname{len} q$. Then $p+q$ is first-column-of-circulant.

(38) If len $p=\operatorname{len} q$ and $p$ is first-column-of-circulant and $q$ is first-column-ofcirculant, then $\operatorname{CCirc}(p+q)=\operatorname{CCirc} p+\operatorname{CCirc} q$.

(39) If $n>0$, then $I_{K}^{n \times n}$ is column circulant.

(40) If $n>0$, then $I_{K}^{n \times n}$ is line circulant.

(41) If $p$ is first-line-of-circulant, then $a \cdot p$ is first-line-of-circulant.

(42) If $p$ is first-line-of-circulant, then $\operatorname{LCirc}(a \cdot p)=a \cdot \operatorname{LCirc} p$.

(43) If $p$ is first-line-of-circulant, then $a \cdot \operatorname{LCirc} p+b \cdot \operatorname{LCirc} p=\operatorname{LCirc}((a+b) \cdot p)$.

(44) If $p$ is first-line-of-circulant and $q$ is first-line-of-circulant and len $p=\operatorname{len} q$ and len $p>0$, then $a \cdot \operatorname{LCirc} p+a \cdot \operatorname{LCirc} q=\operatorname{LCirc}(a \cdot(p+q))$.

(45) If $p$ is first-line-of-circulant and $q$ is first-line-of-circulant and len $p=$ len $q$, then $a \cdot \operatorname{LCirc} p+b \cdot \operatorname{LCirc} q=\operatorname{LCirc}(a \cdot p+b \cdot q)$.

(46) If $p$ is first-column-of-circulant, then $a \cdot p$ is first-column-of-circulant.

(47) If $p$ is first-column-of-circulant, then $\operatorname{CCirc}(a \cdot p)=a \cdot \operatorname{CCirc} p$.

(48) If $p$ is first-column-of-circulant, then $a \cdot \operatorname{CCirc} p+b \cdot \operatorname{CCirc} p=\operatorname{CCirc}((a+$ $b) \cdot p)$.

(49) Suppose $p$ is first-column-of-circulant and $q$ is first-column-of-circulant and len $p=\operatorname{len} q$ and len $p>0$. Then $a \cdot \operatorname{CCirc} p+a \cdot \operatorname{CCirc} q=\operatorname{CCirc}(a$. $(p+q))$. 
(50) If $p$ is first-column-of-circulant and $q$ is first-column-of-circulant and len $p=\operatorname{len} q$, then $a \cdot \operatorname{CCirc} p+b \cdot \operatorname{CCirc} q=\operatorname{CCirc}(a \cdot p+b \cdot q)$.

Let $K$ be a set and let $M$ be a matrix over $K$. We introduce $M$ is circulant as a synonym of $M$ is line circulant.

\section{Some Properties of Anti-Circular Matrices}

Let $K$ be a field, let $M_{1}$ be a matrix over $K$, and let $p$ be a finite sequence of elements of $K$. We say that $M_{1}$ is anti-circular about $p$ if and only if the conditions (Def. 9) are satisfied.

(Def. 9)(i) $\quad$ len $p=$ width $M_{1}$,

(ii) for all natural numbers $i, j$ such that $\langle i, j\rangle \in$ the indices of $M_{1}$ and $i \leq j$ holds $\left(M_{1}\right)_{i, j}=p(((j-i) \bmod \operatorname{len} p)+1)$, and

(iii) for all natural numbers $i, j$ such that $\langle i, j\rangle \in$ the indices of $M_{1}$ and $i \geq j$ holds $\left(M_{1}\right)_{i, j}=(-p)(((j-i) \bmod \operatorname{len} p)+1)$.

Let $K$ be a field and let $M$ be a matrix over $K$. We say that $M$ is anti-circular if and only if:

(Def. 10) There exists a finite sequence $p$ of elements of $K$ such that len $p=$ width $M$ and $M$ is anti-circular about $p$.

Let $K$ be a field and let $p$ be a finite sequence of elements of $K$. We say that $p$ is first-line-of-anti-circular if and only if:

(Def. 11) There exists a square matrix over $K$ of dimension len $p$ which is anticircular about $p$.

Let $K$ be a field and let $p$ be a finite sequence of elements of $K$. Let us assume that $p$ is first-line-of-anti-circular. The functor ACirc $p$ yields a square matrix over $K$ of dimension len $p$ and is defined by:

(Def. 12) ACirc $p$ is anti-circular about $p$.

One can prove the following propositions:

(51) If $M_{1}$ is anti-circular, then $a \cdot M_{1}$ is anti-circular.

(52) If $M_{1}$ is anti-circular and $M_{2}$ is anti-circular, then $M_{1}+M_{2}$ is anticircular.

(53) Let $K$ be a Fanoian field, $n, i, j$ be natural numbers, and $M_{1}$ be a square matrix over $K$ of dimension $n$. Suppose $\langle i, j\rangle \in$ the indices of $M_{1}$ and $i=j$ and $M_{1}$ is anti-circular. Then $\left(M_{1}\right)_{i, j}=0_{K}$.

(54) If $M_{1}$ is anti-circular and $\langle i, j\rangle \in \operatorname{Seg} n \times \operatorname{Seg} n$ and $k=i+1$ and $l=j+1$ and $i<n$ and $j<n$, then $\left(M_{1}\right)_{k, l}=\left(M_{1}\right)_{i, j}$.

(55) If $M_{1}$ is anti-circular, then $-M_{1}$ is anti-circular.

(56) If $M_{1}$ is anti-circular and $M_{2}$ is anti-circular, then $M_{1}-M_{2}$ is anticircular. 
(57) If $M_{1}$ is anti-circular about $p$ and $n>0$, then $p=\operatorname{Line}\left(M_{1}, 1\right)$.

(58) If $p$ is first-line-of-anti-circular, then $-p$ is first-line-of-anti-circular.

(59) If $p$ is first-line-of-anti-circular, then $\operatorname{ACirc}(-p)=-\operatorname{ACirc} p$.

(60) Suppose $p$ is first-line-of-anti-circular and $q$ is first-line-of-anti-circular and len $p=\operatorname{len} q$. Then $p+q$ is first-line-of-anti-circular.

(61) If $p$ is first-line-of-anti-circular and $q$ is first-line-of-anti-circular and len $p=\operatorname{len} q$, then $\operatorname{ACirc}(p+q)=\operatorname{ACirc} p+\operatorname{ACirc} q$.

(62) If $p$ is first-line-of-anti-circular, then $a \cdot p$ is first-line-of-anti-circular.

(63) If $p$ is first-line-of-anti-circular, then $\operatorname{ACirc}(a \cdot p)=a \cdot \operatorname{ACirc} p$.

(64) If $p$ is first-line-of-anti-circular, then $a \cdot \operatorname{ACirc} p+b \cdot \operatorname{ACirc} p=\operatorname{ACirc}((a+$ $b) \cdot p)$.

(65) Suppose $p$ is first-line-of-anti-circular and $q$ is first-line-of-anti-circular and len $p=\operatorname{len} q$ and len $p>0$. Then $a \cdot \operatorname{ACirc} p+a \cdot \operatorname{ACirc} q=\operatorname{ACirc}(a \cdot$ $(p+q))$.

(66) Suppose $p$ is first-line-of-anti-circular and $q$ is first-line-of-anti-circular and len $p=\operatorname{len} q$. Then $a \cdot \operatorname{ACirc} p+b \cdot \operatorname{ACirc} q=\operatorname{ACirc}(a \cdot p+b \cdot q)$.

Let us consider $K, n$. Observe that $0_{K}^{n \times n}$ is anti-circular.

\section{REFERENCES}

[1] Grzegorz Bancerek and Krzysztof Hryniewiecki. Segments of natural numbers and finite sequences. Formalized Mathematics, 1(1):107-114, 1990.

[2] Czesław Byliński. Functions and their basic properties. Formalized Mathematics, 1(1):5565, 1990.

[3] Czesław Byliński. Partial functions. Formalized Mathematics, 1(2):357-367, 1990.

[4] Czesław Byliński. Some basic properties of sets. Formalized Mathematics, 1(1):47-53, 1990.

[5] Katarzyna Jankowska. Transpose matrices and groups of permutations. Formalized Mathematics, 2(5):711-717, 1991.

[6] Eugeniusz Kusak, Wojciech Leończuk, and Michał Muzalewski. Abelian groups, fields and vector spaces. Formalized Mathematics, 1(2):335-342, 1990.

[7] Karol Pạk. Basic properties of the rank of matrices over a field. Formalized Mathematics, 15(4):199-211, 2007.

[8] Michał J. Trybulec. Integers. Formalized Mathematics, 1(3):501-505, 1990.

[9] Wojciech A. Trybulec. Groups. Formalized Mathematics, 1(5):821-827, 1990.

[10] Zinaida Trybulec. Properties of subsets. Formalized Mathematics, 1(1):67-71, 1990.

[11] Edmund Woronowicz. Relations and their basic properties. Formalized Mathematics, 1(1):73-83, 1990.

[12] Xiaopeng Yue, Xiquan Liang, and Zhongpin Sun. Some properties of some special matrices. Formalized Mathematics, 13(4):541-547, 2005.

[13] Katarzyna Zawadzka. The sum and product of finite sequences of elements of a field. Formalized Mathematics, 3(2):205-211, 1992.

[14] Katarzyna Zawadzka. The product and the determinant of matrices with entries in a field. Formalized Mathematics, 4(1):1-8, 1993. 